\title{
TIPS CREATION IN PATIENTS WITH PERSISTENT LEFT SUPERIOR VENA CAVA
}

\author{
Vendelín Chovanec ${ }^{1}$, Antonín Krajina ${ }^{1}$, Petr Hůlek ${ }^{2}$, Miroslav Měštan ${ }^{3}$, Ondřej Renc ${ }^{1}$ \\ Charles University in Prague, Faculty of Medicine and University Hospital Hradec Králové, Czech Republic: Department \\ of Radiology ${ }^{1}, 2^{\text {nd }}$ Department of Internal Medicine, Division of Gastroenterology ${ }^{2}, 1^{\text {st }}$ Department of Internal Medicine ${ }^{3}$
}

\begin{abstract}
Summary: Transjugular intrahepatic portosystemic shunt is a minimally invasive endovascular procedure that has played an important role in the treatment of acute or repeated variceal bleeding or refractory ascites. The standard venous access route for this procedure is the right jugular vein. Sometimes it is better to use the left jugular vein because of lower probability of life threatening complication or technical failure. In this case reports the authors have described their experience with TIPS creation in two patients with persistent left and absent right superior vena cava and recommend using the left jugular vein as an access route in this rare anatomical variant.
\end{abstract}

Key words: Left superior vena cava; Congenital anomaly; Venous access; Transjugular intrahepatic portosystemic shunt

\section{Introduction}

Transjugular intrahepatic portosystemic shunt (TIPS) is an accepted method for treatment of symptomatic portal hypertension especially in the management of uncontrolled or repeated variceal bleeding in spite of endoscopic therapy or in the management of refractory ascites. TIPS is a minimally invasive procedure and the right internal jugular vein (IJV) is recommended as a standard venous access, for there is lower risk of mediastinal injury than using the left IJV (5). The left jugular approach is used in patients in whom the right IJV is thrombosed and can not be cannulated, or portal vein access is unsatisfactory or unsuccessful via the right IJV due to horizontal orientation of the hepatic vein (5). We report successful TIPS creation via the left IJV in two patients with the congenital central venous anomaly such as persistent left superior vena cava (LSVC) with absent right superior vena cava.

\section{Case reports}

Case 1.

A 64-year-old female with cryptogenic liver cirrhosis Child-Pugh class $\mathrm{C}$ and refractory ascites was admitted to our hospital for TIPS creation in April 1994. She had no previous episode of gastroesophageal variceal bleeding. The physical examination revealed large ascites, umbilical hernia, Caput Medusae, and bilateral perimaleolar edema. Computed tomography confirmed ultrasonographic findings of the shrunken liver, splenomegaly, massive ascites with an umbilical hernia, and large portosystemic collaterals via the coronary vein and short gastric veins. The protrombine time was elevated at $18.2 \mathrm{sec}$, total bilirubin $25 \mu \mathrm{mol} / \mathrm{L}$, albumin $27.8 \mathrm{~g} / \mathrm{L}$. An attempt to introduce a Swan-Ganz catheter, in order to perform hemodynamic measurement, via the left subclavian vein failed. The catheter was left in the left subclavian vein and the patient was transferred to the angiographic suite, because of the suspicion of the SVC anomaly - the left sided SVC above all. Phlebography performed through the catheter confirmed the LSVC draining into the right atrium via hypertrophic coronary sinus. After this, the right femoral vein was punctured and the catheter was passed through the dilated coronary sinus into the left IVJ (Fig. 1A) and straightened by a very stiff guidewire (J Back-up Meier, Boston Scientific/Meditech, Watertown, USA). The patient tolerated this manoeuvre without dysrhythmia, and this confirmed the possibility of TIPS creation using the left IJV approach. TIPS procedure was performed 5 days after the diagnostic angiogram with of a surgeon and anesthesiologist present in view of the potential risk of cardiac or large vessel injury. After gaining left IJV access, a soft guidewire (Bentson, Cook Europe, Bjaeverskov, Denmark) and 5F catheter was advanced via the LSVC and coronary sinus into the inferior vena cava. The soft wire was replaced by an extra stiff guidewire over which the 10F sheath of TIPS set (TIPSI-100, Cook Europe, Bjaeverskov, Denmark) was inserted. The rigid steel cannula was advanced into the right hepatic vein. After several unsuccessful needle passes, with one of them complicated by the puncture of the liver capsule, the guidewire 


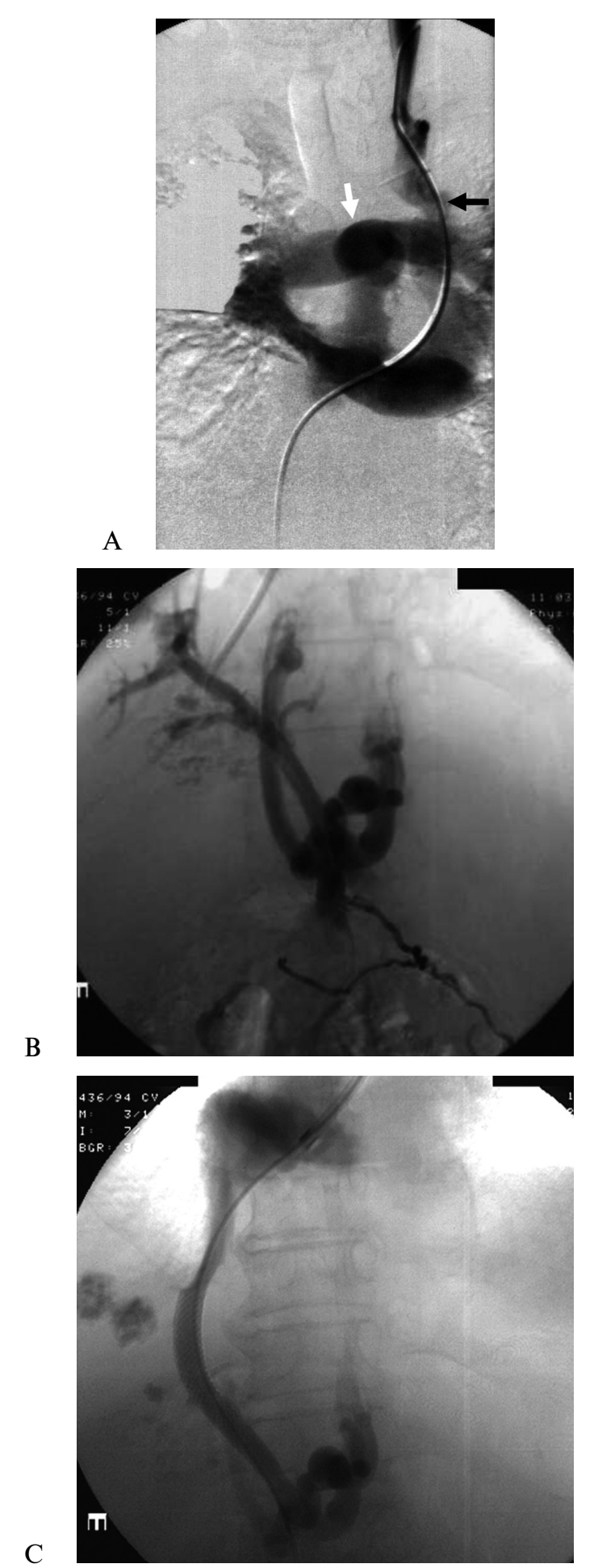

Fig. 1: A) Venogram through catheter in the left jugular vein introduced from the right femoral approach. LSVC (black arrow) and pulmonary artery (white arrow) are opacified by iodinated contrast media. B) Initial transjugular portography shows large portosystemic collaterals and patent portal vein. C) Final portogram after TIPS creation with selfexpandable stent.

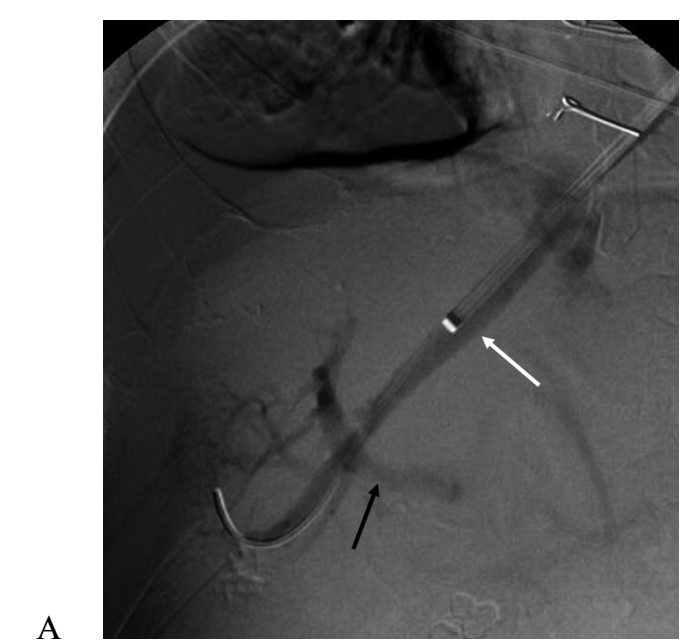

A

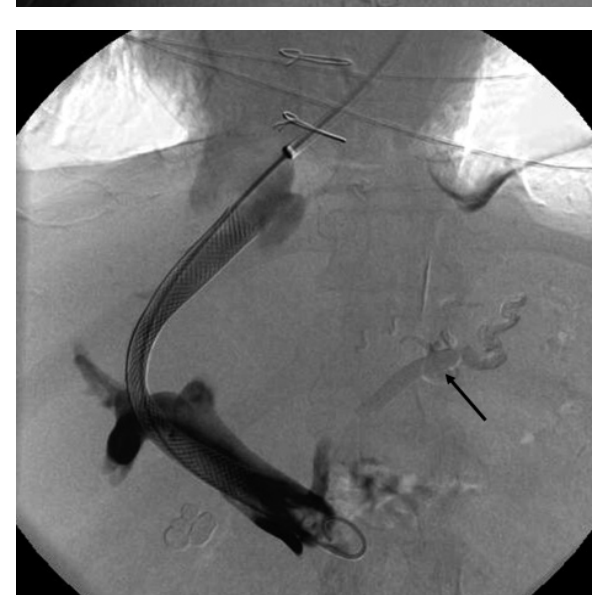

B

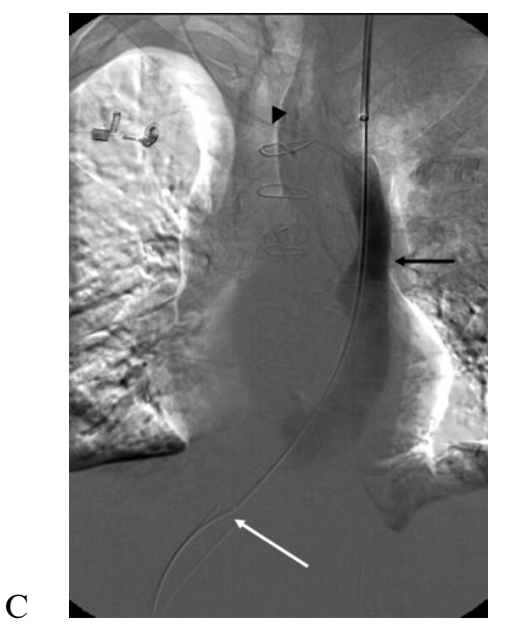

Fig. 2: A) Carbon dioxide wedged hepatic venogram. The right branch of portal vein is marked with black arrow and the middle hepatic vein with white arrow. B) Final portal venogram after coronary vein embolization (black arrow) and shunt creation. C) Phlebography through the retracted sheath with extra stiff wire left in the cranial end of TIPS (white arrow). Black arrow marks persistent LSVC which enters the right atrium via dilated coronary sinus. Black arrowhead shows central venous catheter. 
entered into the branch of the right portal vein. Direct portography revealed varices filled via coronary and short gastric veins (Fig. 1B). The TIPS was created using $10 \mathrm{~mm}$ x $70 \mathrm{~mm}$ selfexpandable stent (Wallstent-Uni, Boston Scientific Corporation, Natick, USA) (Fig. 1C). Portal pressure fell down from $38 \mathrm{mmHg}$ to $27 \mathrm{mmHg}$ and portosystemic gradient decreased from $21 \mathrm{mmHg}$ to $10 \mathrm{mmHg}$. Portosystemic collaterals were not opacified at the digital portogram after the stent placement. Postprocedural course was without complication. Doppler sonography 6 days after the procedure demonstrated a patent shunt with good function and the patient was discharged. Unfortunately she died of an unrelated myocardial infarction one month later.

\section{Case 2.}

A 66-year-old man with portal hypertension and repeated variceal hemorrhage in spite of endoscopic sclerotherapy was admitted to another hospital for TIPS creation in March 2009. The last episode of variceal bleeding led to hemorrhagic shock. Portal hypertension was secondary to alcoholic cirrhosis. The patient was class $\mathrm{C}$ according to Child-Pugh classification and he suffered from chronic heart failure with $30 \%$ ejection fraction. The radiologist cannulated the right IJV and he performed phlebography because he could not introduce guidewire into SVC. Phlebography revealed the LSVC drained into the right atrium through a hypertrophic coronary sinus. The right SVC was absent. The procedure was abandoned because of the high risk of mediastinal injury during introducing stiff metallic cannula of TIPS set in the hepatic vein. The patient was transferred to our institution. The second procedure was performed 2 days after the first attempt. In our experience of this anomaly, the course of guidewire from left IJV to the hepatic vein through this anomaly is less curved than from the right IJV and so interventional radiologist selected the left IJV as an access site. Curved catheter was introduced into the middle hepatic vein and $10 \mathrm{~F}$ sheath was pushed in the hepatic vein over the extra stiff guidewire (Amplatz super stiff, Boston Scientific Corporation, Natick, USA). Carbon dioxide wedged portography was performed (Fig. 2A) and the rigid metallic cannula of TIPS set (TIPSI-100, Cook Europe, Bjaeverskov, Denmark) was inserted in the hepatic vein over extra stiff guidewire. The right branch of portal vein was punctured at the fourth attempt and carbon dioxide portography through the pigtail catheter was performed. Direct portography depicted portosystemic collaterals rising from the coronary vein. The coronary vein was embolized with mixture of n-butyl 2-cyanoacrylate (Histoacryl, B Braun Aesculap, Tuttlingen, Germany) and oil contrast media (Lipiodol Ultrafluide, Guerbert, Ceres, France). The shunt was created using 12 mm x 90 mm selfexpandable stent (Wallstent-Uni, Boston Scientific Corporation, Natick, USA) which was dilated with $8 \mathrm{~mm}$ balloon (Fig. 2B). Portosystemic gradient decreased from $19 \mathrm{mmHg}$ to $10 \mathrm{mmHg}$. At the end of the procedure the sheath was retracted to the left IJV and phlebography showed no extravasation (Fig. 2C). Ultrasound on the first postoperative day a revealed patent shunt and the patient was transferred back to the referring hospital. Five months after procedure he lives with patent shunt and no symptoms of portal hypertension.

\section{Discussion}

The right IJV is the standard access route for TIPS procedure because the IJV, brachiocephalic vein, SVC on the right side and right atrium form a straight channel which is more suitable for manipulation with a stiff cannula during procedure than the left sided approach. Any congenital anomaly of these veins can cause technical problems and TIPS creation may be difficult or impossible via the right IJV.

Congenital anomalies of the SVC are rare with an incidence in general population $0.3-0.5 \%$ (2). The most common of them is the LSVC. Two types of the LSVC have been described - the first one is associated with normal location of visceral organs and the LSVC usually enters the heart via dilated coronary sinus. The second one is associated with complete situs viscerum inversus and enters directly in the morphologic right atrium lying on the left side. Successful TIPS placement has been described in both type of the LSVC (1, 3). Between September 1992 and August 2009 we found 3 cases of the SVC anomalies in our group of 815 patients $(0.4 \%)$ who underwent TIPS placement. Two patiens had first type of LSVC and one had situs viscerum inversus.

The incidence of persistent LSVC is about $0.3 \%$ in general population and $4.4 \%$ in patients with congenital cardiac disease (1). The LSVC is the most common anomaly of systemic vein circulation. It is associated with patent right SVC in $82-90 \%$ (double superior vena cava) and absence of the left brachiocephalic vein in $65 \%$ of patients. Nearly all the LSVC (in $92 \%$ cases) enter the right atrium via the coronary sinus. The rest drains into the left atrium, hepatic vein or inferior vena cava. Drainage to the left atrium cause right to left shunting, which can lead to paradoxical embolisation (7). The anomaly is due to failed obliteration of the left anterior cardinal vein. The first explanation for embryonic development of the persistent left SVC was described by Marshall in 1850 (10).

Increasing number of multidetector row computed tomography (MDCT) and magnetic resonance (MR) imaging including angiography, central venous cannulation, endovascular venous procedures such as pacemaker, defibrilator leads implantation, vena cava filter implantation, TIPS creation or transjugular biopsy has contributed to more frequent detection of LSVC during the life of a patient. This anomaly is rare, but an operator must be able to modify a procedure when it is discovered. The diagnosis can be anticipated by signs on chest X-ray, such as paramediastinal strip, paramediastinal bulging below the aortic arch, widening of aortic shadow, crescent along the left heart border in persistent LSVC $(6,14)$ or revealed by noninvasive methods 
like MDCT or MR angiography (15). There are two reports describing successful TIPS placement and inferior vena cava filter placement through the jugular access $(2,11)$ and one article of unsuccessful transjugular biopsy due to persistent LSVC (4). The authors used the right IJV for implantation of vena cava filter. From the right side there is a longer distance between the infrarenal part of vena cava and jugular vein. This condition allows a gentle curvature of a long introducer sheath, hence it is possible to use either the right or left jugular vein in this case (11).

The primary use of the left IJV approach was chosen in these cases, because this allowed easier cannulation of the hepatic vein. There is a higher risk of dysrhythmia, retrosternal pain, cardiac arrest with persistent LSVC because devices are introduced into the hepatic vein through heart causing distortion of the coronary sinus and right atrium (8).

The right IJV vein approach is preferred for the following reasons: the right IJV continues in a straight line with the SVC down to the inferior vena cava facilitating easy manipulation. The thoracic duct is on the left side, the apex of the right lung is lower in most cases than the left one reducing the risk of procedural comlications. The course from the left side through the left brachiocephalic vein to the SVC is angled. This may cause the thoracic pain from stretching of the mediastinal vessels with the stiff metallic cannula. Hausegger at al. (5) found the left side approach helpful in patients with horizontal course of the right hepatic vein and in the patients with cranially located the right portal vein. The stiff cannula takes a diagonal course through the mediastinum and reaches the right hepatic vein at a more obtuse angle which can facilitate positioning and stabilisation of the metallic cannula in this vein.

We have described two cases of a rare congenital central venous anomaly in patients who underwent successful TIPS procedure using a simple modification of the routine technique. Others have described different techniques of TIPS creation such as the femoral, transmesenteric or transhepatic approach. According to our experience these approaches should be considered much more complicated in cases with the persistent left SVC and absent right SVC $(9,12,13)$. Based on our experience we conclude that the access via left jugular vein should be considered as the method of choice in liver interventions including transjugular liver biopsy and TIPS in the presence of a congenital central venous anomaly such as persistent LSVC with absent right superior vena cava.

\section{References}

1. Bahramipour P, Abu-Judeh HH, Miller J. Transjugular intrahepatic portosystemic shunt placement in a patient with absent right superior vena cava and persistent left superior vena cava. AJR 1999;173:631-2.

2. Campbell M, Deuchar DC. The left-sided superior vena cava. Br Heart J 1954; $16: 423-439$.

3. Chovanec V, Krajina A, Lojík M, Hůlek P, Vaňásek T. TIPS creation in a patient with situs inversus totalis. Cardiovasc Intervent Radiol 2002;25:447-9.

4. Druart F, Cosse P, Aboul-Hosn H, Carre J1, Guyot G. Left superior vena cava: a cause of failure of transjugular liver biopsy and difficulty inserting a pacemaker catheter. Nouv Presse Med 1981;10:36-45.

5. Hausegger KA, Tauss J, Karaic K, Klein GE, Uggowitzer M. Use of the left internal jugular vein approach for transjugular portosystemic shunt. AJR 1998; $171: 1637-9$

6. Higgs AG, Paris S, Potter F. Discovery of left-sided superior vena cava during central venous catheterization. Br J Anaesth 1998;81:260-1.

7. Köcher K, Černá M, Hutyra M, Novotný J, Kozák J, Buřval S. Persistent left superior vena cava- possible source of the paradoxical embolisation. Ces Radiol 2009;63:129-32.

8. Krajina A, Lojík M. TIPS - Technique. In Hůlek P, Krajina A: Current practice of TIPS. Olga Štambergová Publishing House, Hradec Králové 2001, 51-119.

9. LaBerge JM, Ring EJ, Gordon RL. Percutaneous intrahepatic portosystemic shunt created via a femoral vein approach. Radiology 1991;181:679-81.

10. Marshall J. On the development of the great anterior thoracic veins in man and mammalia: including an account of certain remnants of fetal structure found in the adult, a comparative view of these great veins in the different mammalia, an analysis of their occasional peculiarities in the human subject. Philos Trans R Soc Lond 1850;140:133-69.

11. Nair S, Ettles D, Robinson G, Scott P. Inferior vena cava filter from left sided superior vena cava. CardioVasc Intervent Radiol 2008;31:S171-3.

12. Raza SA, Walser E, Hernandez A, Chen K, Marroquin S. Transhepatic puncture of portal and hepatic veins for TIPS using a single-needle pass under sonographic guidance. AJR 2006;187:W87-92.

13. Rozenblit G, Del Guercio LR, Savino JA, et al. Transmesenteric-transfemoral method of intrahepatic portosystemic shunt placement with minilapparotomy. J Vasc Interv Radiol 1996; 7:499-506.

14. Spearman P, Leier CV. Persistent left superior vena cava, unusual wave countour of left jugular vein as the presenting feature. Am Heart J 1990;120:999-1002.

15. White CS, Baffa JM, Haney PJ, Pace ME, Campbell AB. MR imaging of congenital anomalies of the thoracic veins. RadioGraphics 1997;17:595-608.

Received: 02/11/2009. Accepted in revised form: 17/12/2009.

\section{Corresponding author:}

Vendelín Chovanec, MD, Ph.D., University Hospital, Department of Radiology, Sokolská 581, 50005 Hradec Králové, Czech Republic; e-mail: chovanec.v@seznam.cz 